\title{
PERFIL DE APTIDÃO FÍSICA RELACIONADA À SAÚDE EM ADOLESCENTES
}

\section{Andressa Ribeiro Contreira \\ Doutoranda pelo Programa de Pós-graduação Associa- do em Educação Física UEM/UEL, Paraná, Brasil. \\ E-mail: andressacontreira@gmail.com \\ Gislaine Contessoto Pizzo}

Mestranda pelo Programa de Pós-graduação Associado em Educação Física UEM/UEL, Paraná, Brasil.

\section{Francielli Ferreira da Rocha}

Mestranda pelo Programa de Pós-graduação Associado em Educação Física UEM/UEL, Paraná, Brasil.

\section{Tatyanne Roiek Lazier-Leão}

Mestranda pelo Programa de Pós-graduação Associado em Educação Física UEM/UEL, Paraná, Brasil.

\section{Aryelle Malheiros Caruzzo}

Graduada em Educação Física pela Universidade Estadual de Maringá (UEM), Maringá (PR), Brasil.

\section{Fernando Copetti}

Doutor em Ciências do Movimento Humano pela Universidade Federal de Santa Maria (UFSM); Docente Associado na Universidade Federal de Santa Maria (UFSM), Brasil.

\section{Thais Silva Beltrame}

Doutora em Ciências do Movimento Humano pela Universidade Federal de Santa Maria (UFSM); Docente da Universidade do Estado de Santa Catarina (UDESC), Brasil.

\begin{abstract}
RESUMO: Objetivou-se investigar o perfil de aptidão física relacionada à saúde de adolescentes de Florianópolis (SC). Participaram 108 adolescentes com 11,31 $\pm 0,54$ anos, de ambos os sexos, de uma escola pública de Florianópolis (SC). Foram avaliadas a função cardiorrespiratória, força/resistência abdominal, flexibilidade e Índice de Massa Corporal (IMC). Os dados foram analisados pelo Teste Quiquadrado $\left(\mathrm{X}^{2}\right)$ ou Exato de Fisher $(p<0,05)$. A maioria dos adolescentes (75,7\%) obteve classificação saudável na flexibilidade, com associação para o sexo feminino $(p=0,043)$, enquanto que na força/resistência abdominal $67,9 \%$ foram classificados na zona de risco, com tendência para meninos $(p=0,004)$. Na função cardiorrespiratória todos os adolescentes foram classificados na zona de risco e para o IMC a maioria foi considerada saudável. Os adolescentes de Florianópolis (SC) mostraram-se aptos nas avaliações de IMC e flexibilidade, com uma tendência de risco para meninos na flexibilidade. Para resistência abdominal e cardiorrespiratória o perfil dos adolescentes foi de risco, independente do sexo.
\end{abstract}

PALAVRAS-CHAVE: Adolescente; Aptidão física; Saúde.

\section{PHYSICAL CAPACITY RELATED TO HEALTH IN ADOLESCENTS}

ABSTRACT: Physical aptitude related to adolescents' health in Florianópolis SC Brazil is investigated. One hundred and eight adolescents, aged $11.31 \pm 0.54$ years, males and females, from a public school in Florianópolis SC Brazil, participated by evaluating their cardio-respiratory function, abdominal strength/resistance, flexibility and Body Mass Index (BMI). Data were analyzed by $\chi^{2}$ test or Fisher exact test $(\mathrm{p}<0.05)$. Most adolescents $(75.7 \%)$ had a good classification for flexibility, associated to females $(p=0.043)$, whereas $67.9 \%$ were classified within the risk zone in the abdominal strength/ resistance, with a trend for males $(\mathrm{p}=0.004)$. In the case of the cardiorespiratory function, all adolescents were classified within the risk zone whereas most were healthy for MBI. Adolescents evaluated were apt for MBI and flexibility evaluation with a risk trend for males in flexibility. Adolescents' profile for abdominal and cardio-respiratory resistance was within a risk range, regardless of gender.

KEY WORDS: Physical capacity; Health; Adolescents. 


\section{INTRODUÇÃO}

Pesquisas têm evidenciado a relação positiva entre atividade física, aptidão física e saúde para crianças e adolescentes (GLANER, 2005; PETROSKI; SILVA; RODRIGUES, 2011) e, por essa razão, a adoção de um estilo de vida apropriado ao desenvolvimento da aptidão física por intermédio da prática de exercício é indicada. Apesar dessas recomendações, estudos nacionais e internacionais têm verificado, ao longo das últimas décadas, que os níveis de atividade física para todas as faixas etárias são baixos e insatisfatórios (BURGOS; REUTER; TORNQUIST, 2012; SUZUKI; MORAES; FREITAS, 2011). Ainda, estes podem ser influenciados pelas transformações tecnológicas que contribuem para a adoção de comportamentos sedentários e aumento das doenças hipocinéticas (DUMITH et al., 2010).

É importante ressaltar que níveis de atividade física gradualmente reduzidos resultam em menor energia despendida, aumento dos riscos de obesidade e doenças que podem permanecer na vida adulta (FAUGHT et al., 2005). Dessa forma, a identificação dessas variáveis na população infantil e adolescente é preocupante e considerada um problema de saúde pública, uma vez que para um bom funcionamento orgânico estão relacionados os componentes de desenvolvimento, crescimento físico e aptidão física (SILVA; PACCINI; GLANER, 2007)

Nessa perspectiva, torna-se necessário conhecer os aspectos de saúde, por meio da avaliação dos componentes de Aptidão Física Relacionada à Saúde (APFRS) (função cardiorrespiratória, força/resistência abdominal, flexibilidade e composição corporal) como meios acessíveis para identificação de casos em que os critérios de saúde não são alcançados. Estudos têm verificado resultados para resistência cardiorrespiratória abaixo do ponto de corte considerado saudável em crianças e adolescentes (BERGMANN et al., 2005; GUEDES, 2002; HOBOLD, 2003; PELEGRINI et al., 2012; SALES-NOBRE; KREBS; VALENTINI, 2009). Nos testes de força/resistência abdominal as pesquisas têm revelado resultados distintos em relação ao atendimento ou não aos critérios de saúde para esta população (BERGMANN et al., 2005; GLANER, 2005; GUEDES, 2002; PELEGRINI et al., 2012).

Em relação à flexibilidade, estudos apontam resultados diversos quanto ao atendimento dos critérios de saúde, não havendo uma evidência de que crianças e adolescentes não atendam aos critérios de saúde nesta avaliação (HENKES, 2013; MASCARENHAS et al., 2013; PELEGRINI et al., 2012; PELEGRINI et al., 2012). Em se tratando das medidas de composição corporal os achados são preocupantes, tendo em vista a verificação da prevalência elevada de excesso de peso para grupos diferentes ao longo dos anos (DINIZ et al., 2005; FARIAS; PETROSKI, 2003; OGDEN et al., 2006; SALOMONS; RECH; LOCH, 2007; SILVA et al., 2005).

A partir das informações apresentadas, observase uma variedade de pesquisas investigando as variáveis de aptidão física na população infantil e adolescente, contudo nota-se que utilizam de critérios de referência nem sempre validados para as características da população brasileira. Diante desses aspectos e considerando a escola como espaço estratégico no desenvolvimento de ações em saúde, este estudo objetivou investigar o perfil de aptidão física relacionada à saúde de adolescentes de Florianópolis (SC), segundo as referências da bateria PROESP/BR.

\section{METODOLOGIA}

A seleção dos participantes foi não probabilística e ocorreu em um universo de aproximadamente 590 alunos, de ambos os sexos, com idades entre 11 e 13 anos de uma escola da rede estadual de ensino localizada na região central de Florianópolis (SC). Esta instituição de ensino é considerada a maior escola pública da América Latina. Obteve-se autorização da equipe pedagógica para fazer o convite a 400 alunos, aos quais foram entregue o Termo de Consentimento Livre e Esclarecido, obtendo-se o retorno de 130 termos assinados pelos pais.

Os critérios de inclusão considerados foram: participação voluntária dos alunos; faixa etária estabelecida; apresentação da autorização dos pais ou responsáveis por meio da assinatura do Termo de Consentimento Livre e Esclarecido; ausência de problemas físicos nos membros inferiores ou superiores e ainda deficiências intelectuais que impossibilitassem a realização das avaliações (indicados pelos professores).

Foram realizadas as avaliações de flexibilidade (sentar e alcançar sem o banco de Wells), força/resistência abdominal (maior número de repetições em 1 minuto), 
função cardiorrespiratória (corrida caminhada de 6 minutos) e Índice de Massa Corporal (IMC), segundo o Manual de Medidas e Avaliações do Projeto Esporte Brasil (GAYA, 2009).

O estudo foi aprovado pelo Comitê de Ética em Pesquisa em Seres Humanos da Universidade do Estado de Santa Catarina (CEPSH/UDESC - parecer $n^{\circ}$ 224/2010). A escola na qual o estudo foi desenvolvido faz parte da Rede de Escolas Estaduais do Município de Florianópolis (SC). Após as reuniões com a direção de ensino para explanação dos objetivos do estudo, foram estabelecidos os turnos para coleta de dados (matutino e vespertino) e indicadas as turmas às quais se poderia ter acesso. As avaliações foram realizadas em ambiente escolar e os alunos foram avaliados individualmente, por avaliadores previamente capacitados e tiveram um período de familiarização com os testes antes do registro dos resultados, conforme descrito nos protocolos. Cada avaliação individual teve duração de 20 minutos, aproximadamente.

Os dados foram analisados no pacote estatístico SPSS versão 17.0 e apresentados por meio da estatística descritiva (frequência simples e percentual) e inferencial. A associação entre a aptidão física em função do sexo dos escolares foi analisada pelo teste não paramétrico $\mathrm{X}^{2} \mathrm{e}$ exato de Fischer com ajuste residual, adotando-se $p<$ 0,05 .

\section{RESULTADOS}

Atendidos os critérios para inclusão no estudo e diante da perda amostral (devido a problemas de saúde, condições adversas do tempo, falta no dia das avaliações e período de greve escolar), o grupo final de participantes foi constituído por 108 escolares (36 meninos e 72 meninas), com média de idade 11,31 ( $\pm 0,54$ anos). As médias de massa corporal e estatura foram $43,50 \mathrm{~kg}$ ( \pm $9,75)$ e $1,50 \mathrm{~cm}( \pm 0,08)$, respectivamente.

Conforme apresentado na Tabela 1, na avaliação do IMC a maioria dos escolares foi classificada na zona saudável (83,3\%). Foi identificado que $75,7 \%$ dos escolares apresentaram classificação na zona saudável no teste de flexibilidade, com associação significativa para o sexo feminino $(p=0,043)$. Por meio do ajuste residual $(2,0)$ observou-se tendência significativa de meninos na classificação zona de risco $(36,1 \%)$ nas medidas de flexibilidade.

$\mathrm{Na}$ avaliação da força/resistência abdominal (Tabela 1), 67,9\% dos escolares foram classificados na zona de risco. Conforme o ajuste residual $(2,9)$ houve tendência significativa de meninos na classificação de risco $(p=0,004)$. Por fim, no teste de avaliação da função cardiorrespiratória nenhum escolar alcançou os critérios da zona de saúde.

Tabela 1. Distribuição de frequência dos escolares nas avaliações da aptidão física relacionada à saúde quanto às zonas de saúde e risco. Florianópolis, 2011

\begin{tabular}{|c|c|c|c|c|c|c|c|}
\hline Avaliações & $\begin{array}{c}\text { Indivíduos } \\
\text { Avaliados** } \\
\text { (n) }\end{array}$ & $\begin{array}{c}\text { Classificação } \\
\text { APFRS }\end{array}$ & $\begin{array}{c}\text { Total } \\
(\mathrm{n}=108)\end{array}$ & $\begin{array}{l}\text { Meninos } \\
(\mathrm{n}=36)\end{array}$ & $\begin{array}{l}\text { Meninas } \\
(\mathrm{n}=72)\end{array}$ & $\mathbf{X}^{2}$ & $P$ \\
\hline & & & $\mathbf{f}(\%)$ & $\mathbf{f}(\%)$ & $\mathbf{f}(\%)$ & & \\
\hline \multirow{2}{*}{ IMC } & \multirow{2}{*}{$(\mathrm{n}=108)$} & ZS & $90(83,3)$ & $28(77,8)$ & $62(86,1)$ & \multirow{2}{*}{1,200} & \multirow{2}{*}{0,273} \\
\hline & & ZR & $18(16,7)$ & $8(22,2)$ & $10(13,9)$ & & \\
\hline \multirow{2}{*}{ Flexibilidade } & \multirow{2}{*}{$(\mathrm{n}=107)$} & ZS & $81(75,7)$ & $23(63,9)$ & $58(81,7)$ & \multirow{2}{*}{4,115} & \multirow{2}{*}{$0,043 *$} \\
\hline & & ZR & $26(24,3)$ & $13(36,1)^{¥}$ & $13(18,3)$ & & \\
\hline \multirow{2}{*}{ Abdominal } & \multirow{2}{*}{$(\mathrm{n}=106)$} & ZS & $34(32,1)$ & $5(13,9)$ & $29(41,4)$ & \multirow{2}{*}{8,276} & \multirow{2}{*}{$0,004 *$} \\
\hline & & ZR & $72(67,9)$ & $31(86,1)^{¥}$ & $40(58,6)$ & & \\
\hline \multirow{2}{*}{ Resist. Cardio. } & \multirow{2}{*}{$(\mathrm{n}=101)$} & ZS & 0 & 0 & 0 & & \\
\hline & & ZR & $101(93,5)$ & 35 (100\%) & $66(100 \%)$ & & \\
\hline
\end{tabular}




\section{DISCUSSÃO}

Com o intuito de investigar a aptidão física relacionada à saúde em adolescentes, os resultados deste estudo revelaram que para o teste de flexibilidade a maioria dos escolares foi classificada na zona saudável, havendo uma tendência de meninos na zona de risco (Tabela 1). Neste componente, resultados positivos para meninas também foram encontrados em outras pesquisas (BURGOS; REUTER; TORNQUIST, 2012; HENKES et al., 2013; MASCARENHAS et al., 2013). Em estudo com escolares de diferentes regióes do Brasil, foram encontrados valores médios superiores na flexibilidade para o sexo feminino em todas as idades analisadas (PELEGRINI et al., 2013). Já para os critérios de saúde os autores verificaram que a maioria dos escolares não atingiu os valores estabelecidos, havendo maior prevalência de meninos na classificação de risco, o que se assemelha ao presente estudo (PELEGRINI et al., 2013). Em pesquisa desenvolvida por Glaner (2005), 40,8\% dos adolescentes não atingiram os critérios estabelecidos para a saúde na flexibilidade, com maior presença de meninas na zona de risco

Os distintos desempenhos entre meninos e meninas nos testes de flexibilidade podem se justificar pelo fato de ocorrer progressos anuais na flexibilidade para meninas até o final da adolescência, de modo que estas diferenças se acentuam durante o estirão de crescimento e maturação sexual (MALINA; BOUCHARD; BAR-OR, 2009). Outro fator que pode contribuir para os desempenhos distintos é o tipo de atividade física incentivada para meninos e meninas, pois exercícios de alongamento são socialmente indicados para meninas e observam-se mais meninas participando de atividades como ginástica e dança (HAYWOOD; GETCHELL, 2010).

Os resultados referentes à força/resistência abdominal constataram que a maioria dos escolares apresentou desempenho na zona de risco, com tendência para os meninos nesta classificação (Tabela 1). Estes resultados são corroborados por pesquisa de Pelegrini et al. (2011) na qual mais de 50\% dos escolares foram classificados na zona de risco.

Já em estudo com escolares paranaenses (HOBOLD, 2003), foram encontrados índices satisfatórios noteste deforça/resistênciaabdominal paraambosossexos utilizando os critérios de referência da FITNESSGRAM. Diferenças na força/resistência abdominal são observadas em todas as idades com melhor desempenho para meninos em relação às meninas, e durante o estirão da adolescência eles obtêm ganhos mais rápidos na força/ resistência abdominal devido ao aumento dos níveis de testosterona (MALINA; BOUCHARD; BAR-OR, 2009). Os autores acrescentam que há um aumento linear nesta capacidade em meninos dos 6 aos 13 anos, os quais posteriormente apresentam um desenvolvimento um pouco acelerado, enquanto que em meninas há um aumento na força/resistência abdominal com a idade até os 14 anos, sem melhora subsequente em média.

Ao comparar os desempenhos no teste de força/resistência abdominal em função do sexo dos adolescentes, nota-se que houve maiores ocorrências de meninas na zona saudável. Este quadro pode ocorrer pelo fato de que elas também apresentaram melhor desempenho no teste de flexibilidade, ou ainda pelo tipo de atividades físicas e motoras que estão engajadas. Esses resultados podem se justificar pelo fato de que a força abdominal é um fator determinante no desempenho do teste sentar e alcançar, uma vez que músculos abdominais fortes puxam o tronco para frente, possibilitando um maior grau de flexão (MALINA; BOUCHARD; BAR-OR, 2009).

Os resultados referentes ao IMC indicaram que a maioria dos adolescentes foi classificada na zona saudável (Tabela 1) e não esteve associado ao sexo. Esse resultado é considerado positivo, ao considerar os altos índices de sobrepeso e obesidade apontados pela literatura. As investigações realizadas por alguns autores (BURGOS; REUTER; TORNQUIST, 2012; GUEDES, 2002) vão ao encontro dos achados dessa pesquisa, indicando IMC de adolescentes de ambos os sexos adequados para sua faixa etária.

Com relação aos testes de função cardiorrespiratória, todos os escolares avaliados foram classificados na zona de risco, o que é considerado preocupante para as questões relativas à saúde. Os dados são confirmados por outras pesquisas avaliando a população infantil e adolescente, as quais também encontraram resultados insatisfatórios para a condição 
cardiorrespiratória (HENKES et al., 2013; PELEGRINI et al., 2012; PETROSKI; SILVA; RODRIGUES, 2011; SALES-NOBRE; KREBS; VALENTINI, 2009) A partir do quadro apresentado, observa-se um estado de alerta quanto às condições cardiorrespiratórias de crianças e adolescentes, uma vez que este componente da aptidão física tem maiores implicações para a saúde, pois se refere à capacidade das pessoas de sustentar a realização de atividades vigorosas (HAYWOOD; GETCHELL, 2010).

Diversos são os fatores que podem influenciar o desempenho na função cardiorrespiratória, dentre eles o estirão de crescimento da adolescência, a diferença entre os sexos na capacidade de trabalho, tamanho corporal, composição corporal, os níveis de hemoglobina, assim como o estilo de vida sedentário, que em qualquer idade leva ao declínio dos sistemas cardíaco, respiratório e vascular (HAYWOOD; GETCHELL, 2010). No entanto, a mudança nos hábitos de vida na população infantil e adolescente pode contribuir para a melhora nas condições relativas à saúde, além de auxiliar na redução dos fatores de risco.

Conforme a literatura, mesmo que meninos e meninas obtenham resultados negativos nas medidas de APFRS ambos têm capacidade de alcançar melhores desempenhos, desde que participem ativamente de atividade física regular, já que as características de desempenho apresentadas por estes na adolescência são influenciados por fatores anatômicos, fisiológicos e também pelos fatores socioculturais (GALLAHUE; OZMUN, 2005). Para este último, os autores enfatizam a formação, educação, bem como as oportunidades de vivências motoras que contribuem de maneira significativa para a adoção de estilos de vida ativos benéficos à saúde.

Observando tais aspectos, reflete-se sobre a importância da atuação dos professores de educação física, no sentido de estimular os alunos durante as aulas de educação física para a manutenção das práticas de atividades física e hábitos alimentares saudáveis, a fim de que esses hábitos sejam agregados em outras fases da vida.

Como limitações do estudo destacam-se o não controle das atividades extracurriculares realizadas pelos adolescentes, bem como a não avaliação da maturação sexual dos escolares as quais poderiam influenciar no desempenho dos testes realizados.
As implicações práticas do presente estudo podem ser verificadas no incentivo às avaliações em saúde no contexto escolar, tendo em vista que neste espaço é possível desenvolver ações e intervenções pontuais visando potencializar a aptidão física relacionada à saúde dos escolares. Sugere-se para futuras pesquisas a avaliação dos aspectos relacionados à maturação sexual e práticas de atividades extracurriculares, as quais podem influenciar os componentes de aptidão física relacionada à saúde dos adolescentes.

\section{CONCLUSÃO}

Os resultados revelaram aspectos satisfatórios quanto ao atendimento dos critérios de saúde para o IMC e flexibilidade para a maioria dos adolescentes, com uma tendência significativa de meninos classificados na zona de risco na flexibilidade. Nas avaliações de força/ resistência abdominal e resistência cardiorrespiratória os resultados foram negativos, principalmente na avaliação cardiorrespiratória, tendo em vista que todos os escolares avaliados, independente do sexo, foram classificados na zona de risco para a saúde.

\section{REFERÊNCIAS}

BERGMANN, G. et al. Aptidão física relacionada à saúde de crianças e adolescentes do Estado do Rio Grande do Sul. Revista Perfil, v. 7, n. 7, p. 12-21, 2005.

BRITO, A. K. A.; SILVA, F. I. C.; FRANÇA, N. M. Programas de intervenção nas escolas brasileiras: uma contribuição da escola para a educação em saúde. Saúde em Debate, v. 36, n. 95, p. 624-632, 2012.

BURGOS, M. S.; REUTER, C. P.; TORNQUIST, L.; PICCIN, A. S.; RECKZIEGEL, M. B.; POHL, H. G. H.; BURGOS, L. T. Health-related physical fitness profile of children and adolescents aged 7-17. J. Health Sci Inst, v. 30, n. 2, p. $171-175,2012$.

DINIZ, I. M. S.; LOPES, A. S.; DUMMEL, C. C. B.; RIEGER, T. Crescimento físico e adiposidade corporal de escolares. Rev Bras Cineantropom Desempenho Hum, v. 8, n. 2, p. 32-38, 2005. 
DUMITH, S. C.; RAMIRES, V. V.; SOUZA, M. J. A.; MORAES, D. S.; PETRY, F. G.; OLIVEIRA, E. S.; RAMIRES, S. V.; MARQUES, A. C. Aptidão física relacionada ao desempenho motor em escolares de 7 a 15 anos. Rev bras educ fís esporte, v. 24, n. 1, p. 5-14, 2010.

FARIAS, E. S.; PETROSKI, E. L. Estado Nutricional e atividade física de escolares da cidade de Porto Velho, RO. Rev Bras Cineantropom Desempenho Hum, v. 5, n. 1, p. 27-38, 2003.

FAUGHT, B. E. et al. Increased risk for coronary vascular disease in children with developmental coordination disorder. J Adolesc Health, v. 37, n. 5, p. 376-380, 2005.

GaLlahue, D. L.; OZMUN, J. C.; GOODWAY, J. D. Compreendendo o desenvolvimento motor: bebês, crianças, adolescentes e adultos. [s.l.]: AMGH, 2013.

GAYA, A. C. A. Manual de aplicação de medidas e testes, normas e critérios de avaliação. Projeto Esporte Brasil - PROESP BRASIL. Porto Alegre. 2009. Disponível em: $<$ http://www.proesp.ufrgs.br> Acesso em: 08 fev. 2013.

GLANER, M. F. Aptidão física relacionada à saúde de adolescentes rurais e urbanos em relação aos critérios de saúde. Rev bras educ fís esporte, v. 19, n. 1, p. 13-24, 2005.

GUEDES, C. Estudo associativo do nível socioeconômico com os hábitos de vida, indicadores de crescimento e aptidão física relacionada à saúde. 2002. 131 f. Dissertação (Mestrado) - Programa de Pós-Graduação em Ciências do Movimento Humano, Universidade Federal do Rio Grande do Sul, Porto Alegre, 2002.

HAYWOOD, K. M.; GETCHELL, N. Desenvolvimento motor ao longo da vida. 6. ed. [s.1.]: Artmed, 2010.

HENKES, C. M.; BORFE, L. B.; MURADÁS, R.; TORNQUIST, L.; BURGOS, M. S. Aptidão física relacionada à saúde de escolares: estudo comparativo dos hemisférios NorteSul-Leste-Oeste da zona rural de Santa Cruz do Sul - RS. Cinergis, v. 14, n. 4, p. 206-209, out./dez. 2013.

HOBOLD, E. Indicadores de aptidão física relacionada à saúde de crianças e adolescentes do município de Marechal Cândido Rondon - Paraná, Brasil. Rev Bras Cineantropom Desempenho Hum, v. 5, n. 1, jul./set. 2003.

MALINA, R. M.; BOUCHARD, C.; BAR-OR, O. Crescimento, maturação e atividade física. São Paulo: Phorte, 2009.

MASCARENHAS, L. P. G.; FERREIRA, A. B. F.; LIMA, V. A.; GRZELCZAK, M. T. Estudo comparativo da aptidão física entre crianças de escola pública e particular: uma visão regional. Cinergis, v. 14, n. 3, p. 157-160, jul./set. 2013.

OGDEN, C. L. et al. Prevalence of overweight and obesity in the United States, 1999-2004. Jama, v. 295, n. 13, p. 1549-1555, 2006. Disponível em: < http:/jama.ama-assn. org/content/295/13/1549.abstract $>$. Acesso em: 12 mar. 2013.

PELEGRINI, A.; SILVA, D. A. S.; PETROSKI, E. L.; GLANER, M. F. Aptidão física relacionada à saúde de escolares brasileiros: dados do Projeto Esporte Brasil. Rev Bras Med Esporte, v. 17, n. 2, 2012. Disponível em: <www. scielo.br/scielo.php/script_sci_serial/pid...pt/nrm_iso $>$ Acesso em: 10 jan. 2013.

PETROSKI, E. L.; SILVA, A. F.; RODRIGUES, A. B.; PELEGRINI, A. Aptidão física relacionada a saúde em adolescentes brasileiros residentes em áreas de médio/ baixo índice de desenvolvimento humano. Rev Salud Pública, v. 13, n. 2, p. 219-228, abr. 2011.

SALES-NOBRE, F. S.; KREBS, R. J.; VALENTINI, N. C. Práticas de lazer, nível de atividade física e aptidão física de moças e rapazes brasileiros. Rev Salud Pública, v. 11, n. 5, p. 713-723, 2009.

SALOMONS, E.; RECH, C. R.; LOCH, M. R. Estado nutricional de escolares de 6-10 anos de idade da rede municipal de ensino de Arapoti, Paraná. Rev Bras Cineantropom Desempenho Hum, v. 9, n. 3, p. 244$249,2007$.

SILVA, D. A. S. et al. Excesso de adiposidade corporal em adolescentes: associação com fatores sóciodemográficos e aptidão física. Motriz, v. 19, n. 1, p. 114-125, 2013. 
SILVA, M. A. M. et al. Prevalência de fatores de risco cardiovascular em crianças e adolescentes da rede de ensino da cidade de Maceió. Arq Bras Cardiol, v. 84, n. 5, p. 387-392, 2005.

SILVA, M. C.; PACCINI, M. K.; GLANER, M. F. Aptidão física relacionada à saúde de adolescentes oriundos de diferentes níveis econômicos. Rev Educ Fís UEM, v. 18, n. 2, p. 199-206, mai. 2007.

SUZUKI, C. S.; MORAES, S. A.; FREITAS, I. C. M. Atividade física e fatores associados em adultos residentes em Ribeirão Preto - SP. Rev Saúde Pública, v. 45, n. 2, p. 311-320, abr. 2011.

Recebido em: 23 de maio de 2016 Versão final recebida em: 01 de julbo de 2016 Aceito em: 03 de julbo de 2016 